\title{
Fractal Simulation of Flocculation Processes Using a Diffusion-Limited Aggregation Model
}

\author{
Dongjing Liu ${ }^{1, *}$, Weiguo Zhou ${ }^{1}$, Zumin Qiu ${ }^{2}$ and Xu Song ${ }^{1}$ \\ 1 College of Mechanical Engineering, Tongji University, Shanghai 200092, China; \\ tjweiguo@tongji.edu.cn (W.Z.); songxu_0612@163.com (X.S.) \\ 2 School of Resources Environmental \& Chemical Engineering, Nanchang University, \\ Nanchang 330031, China; mzqiu@ncu.edu.cn \\ * Correspondence: liudongjing19@163.com; Tel./Fax: +86-021-6598-1482
}

Received: 25 October 2017; Accepted: 15 November 2017; Published: 18 November 2017

\begin{abstract}
In flocculation processes, particulates randomly collide and coagulate with each other, leading to the formation and sedimention of aggregates exhibiting fractal characteristics. The diffusion-limited aggregation (DLA) model is extensively employed to describe and study flocculation processes. To more accurately simulate flocculation processes with the DLA model, the effects of particle number (denoting flocculation time), motion step length (denoting water temperature), launch radius (representing initial particulate concentration), and finite motion step (representing the motion energy of the particles) on the morphology and structure of the two-dimensional (2D) as well as three-dimensional (3D) DLA aggregates are studied. The results show that the 2D DLA aggregates possess conspicuous fractal features when the particle number is above 1000, motion step length is 1.5-3.5, launch radius is $1-10$, and finite motion step is more than 3000; the 3D DLA aggregates present clear fractal characteristics when the particle number is above 500 , the motion step length is $1.5-3.5$, the launch radius is $1-10$, and the finite motion step exceeds 200. The fractal dimensions of 3D DLA aggregates are appreciably higher than those of 2D DLA aggregates.
\end{abstract}

Keywords: fractal; flocculation; diffusion-limited aggregation; DLA; finite motion step

\section{Introduction}

Flocculation is an important method in the field of water and wastewater treatment [1-3]; it can assist in migrating, transforming, and removing particulate matter in water. In flocculation processes, particulates in colloidal suspension randomly collide and coagulate with each other, resulting in the formation and sedimention of fractal-like flocs (aggregates). It is well known that the structure of flocs (aggregates) has a vital impact on the properties of the flocculated colloidal suspension; a few research works have contributed towards the understanding of this impact. Jiang and Logan [4] examined the fractal dimensions of fluorescent microsphere aggregates, and reported that the fractal dimensions of colloidal aggregates are 1.8 and 2.2 when the particles are highly destabilized and more stable, respectively. Oles [5] found that the fractal dimensions increased from 2.1 to 2.5, resulting from the compaction of the aggregate structure which was attributed to the selective breakup that removed the weaker (and more porous) parts of the colloidal aggregates. Adachi et al. [6,7] conducted many valuable works on the structure properties of colloidal flocs, such as effect of ionic strength on the initial flocculation dynamics [8], shear viscosity of coagulated suspensions [9], effect of floc structure on the rate of shear coagulation [10], and the restructuring behavior of small flocs [11].

The diffusion-limited aggregation (DLA) model is an idealization of the process by which matter irreversibly combines to form random objects in the case where the rate-limiting step is the diffusion of matter to the aggregate [12,13]. Therefore, the DLA model is often used to describe flocculation processes, and study of the DLA model could help us to better understand flocculation processes and 
contribute to the development of flocculants and flocculation methods. To date, the DLA model has been extensively studied [14-16] and applied in many fields, such as fluid displacement in porous media [17,18], electroless deposition dynamics of silver nanoparticle clusters [19], and wax/asphaltene aggregation in crude oil [20]. Novotny et al. [21] assumed that the DLA clusters grew with the inclusion of varying amounts of surface tension, and found that the DLA cluster rapidly breaks up into many disconnected, smaller clusters but the system fractal dimension is essentially unchanged. Tan et al. [22] conducted two-dimensional DLA simulations with different particle sizes, and found that when large particles were in the minority, the patterns of clusters appear asymmetrical and nonuniform, and their fractal dimensions increase. Braga et al. [23] studied the influence of particle size on DLA clusters, and proposed an angle selection mechanism on dendritic growth that influences the shielding effect of the DLA edge and affects the fractal dimension of the clusters. Deng [24] performed two-dimensional DLA simulations with various particle shapes (polygons), and reported that different particle shapes only change the local structure but have no impact on the global structure of the aggregates, although the local compactness decreases as the number of polygon edges increases. Xu [25] studied the effects of varying particle motion step length, maximum motion step number, and collision probability on the morphology and structure of the flocculation process by using the two-dimensional DLA model.

Though many studies on the DLA model have been conducted, most of the researchers only focused on one or few factors and their influences on the fractal characteristics of the two-dimensional DLA aggregates. In this work, the we address the impacts of four key model parameters-particle number (denoting flocculation time, $N$ ), motion step length (denoting water temperature, $N_{s}$ ), launch radius (representing initial particulate concentration, $R_{e}$ ), and finite motion step (representing the motion energy of the particles, $M_{s}$ ) - on the morphology, fractal dimensions $\left(D_{f}\right)$, and porosities $\left(V_{f}\right)$ of the two-dimensional (2D) as well as three-dimensional (3D) DLA aggregates, in order to get a deeper understanding of the fractal features of the DLA aggregates, which would assist in describing flocculation processes more exactly.

\section{Model}

The classical DLA simulation is carried out in the following steps [26,27], and depicted in Figure 1a. First, place a fixed "seed" particle (the seed radius is $R_{0}$ ) in the center of a square divided into an $\mathrm{L} \times \mathrm{L}$ grid (or a sphere divided into an $\mathrm{L} \times \mathrm{L} \times \mathrm{L}$ grid) and draw a large circle (or a big sphere) centering around the "seed" particle; the radius of the circle (or the sphere) is the so-called launch radius $\left(R_{e}\right)$. Then, release a free particle (not the "seed" particle) from any site on the perimeter of the circle (or the sphere). The launched particle moves randomly, following a Brownian path, which means that it walks in a random direction at each step, with no correlation to the previous direction, in which the new position of the free particle is given by

$$
\begin{aligned}
& x_{n}=x_{n-1}+\mathrm{R}_{e} \cos \alpha, \\
& y_{n}=y_{n-1}+\mathrm{R}_{e} \sin \alpha, \\
& z_{n}=z_{n-1}+\mathrm{R}_{e} \cos \beta,
\end{aligned}
$$

where $\alpha, \beta$ are angles randomly allocated at each motion step. Assume the free particle movement has two possible results: One is that it arrives at one of the lattice sites adjacent to the occupied sites, then stops and generates another particle; another is that it leaves the circle (or the sphere) and disappears. The killing radius $\left(R_{k}\right)$ is a limiting radius that is used to identify a disposal event when the walking particle moves beyond the maximum circle (or sphere) region. Another particle is then launched and halted when adjacent to the two occupied sites, and so forth. As this process repeats a large number of times, a fractal-like DLA aggregate can be formed.

The "seed" particle and other free particles are considered to have circular shapes (or spherical shapes) and their radius is fixed at 0.5 . The fractal dimensions of the DLA aggregates are obtained 
by using the gyration calculation method $[7,28,29]$. Particularly, the number of primary particles is a function of the gyration radius of the aggregates (depicted in Figure 1b), which obeys the following scaling form:

$$
N=k_{0}\left(\frac{R_{g}}{R_{0}}\right)^{D_{f}}
$$

where $N$ is the number of primary particles enclosed in a circle (or sphere) centered at the aggregate center, $k_{0}$ is the prefactor, $R_{g}$ is the gyration radius denoting the maximum radius of the aggregate, $R_{0}$ is the radius of the primary particle, and $D_{f}$ is the Hausdorff dimension (fractal dimension). Equation (4) in logarithmic form can be expressed as follows:

$$
\log (N)=\log \left(k_{0}\right)+D_{f} \log \left(\frac{R_{g}}{R_{0}}\right) .
$$

If we plot $\log (N)$ against $\log \left(R_{g} / R_{0}\right)$, a straight line can be obtained, and the slope of the fitting curve is equal to the fractal dimension $D_{f}$.

(a)

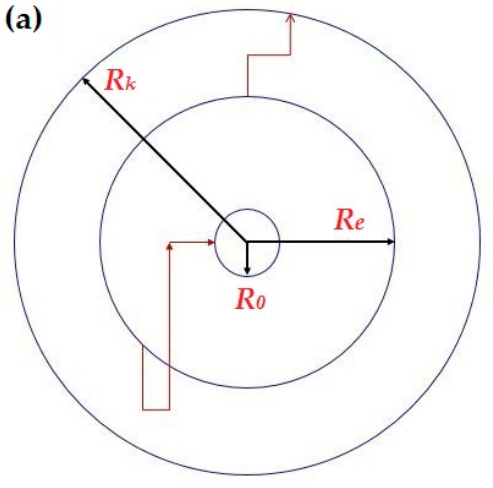

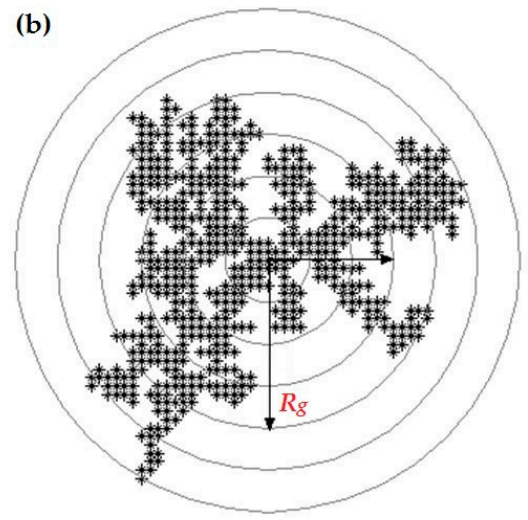

Figure 1. Sketch of particle aggregation of DLA model (a) and fractal dimension calculation by using gyration method (b).

The porosity can be determined using the following formulas:

$$
\begin{gathered}
V_{f}=1-\frac{\pi r_{p}^{2} N}{\pi R_{g}^{2}}=1-N\left(\frac{r_{p}}{R_{g}}\right)^{2} \quad(2 D), \\
V_{f}=1-\frac{\frac{4}{3} \pi r_{p}^{3} N}{\frac{4}{3} \pi R_{g}^{3}}=1-N\left(\frac{r_{p}}{R_{g}}\right)^{3} \quad(3 D),
\end{gathered}
$$

where $r_{p}$ is the radius of the primary particle and $V_{f}$ is the porosity of the DLA aggregate.

\section{Results and Discussion}

\subsection{Effect of Particle Number}

The fractal simulation was conducted with a motion step length of $2\left(N_{s}=2\right)$, a launch radius of $2\left(R_{e}=2\right)$, and different particle numbers; the effect of particle number on the fractal features and structures of the DLA aggregates is shown in Figures 2 and 3. Generally, the particle number relates to the flocculation time: the higher the number of particles, the longer the flocculation time. For the 2D DLA simulation, when the particle number is fairly small $(N<1000)$, the aggregates are fairly small and only show local fractal features, and their scale-invariances as well as self-similarities are insignificant. The aggregate gradually grows with rising particle numbers, and it presents typical 
fractal morphology with properties of global scale-invariances when the particle number reaches a certain value $(N \geq 1000)$. The fractal dimension increases rapidly as the particle number rises from 50 to 1000, and then changes little as the particle number increases further (Figure 4). The fractal dimension slightly fluctuates in the range 1.66-1.74, which coincides with the fractal dimension value range of typical 2D DLA aggregates [12]. The porosity is smaller (around 0.8) when the particle number is less than 500, as it is easier to form a compact DLA aggregate when the particle numbers are lower, and it becomes increasingly compact and grows to a two-dimensional branched aggregate with rising particle numbers, leading to the increase in the fractal dimension and porosity. Therefore, the optimal particle number for 2D DLA simulation is 2000. For 3D DLA simulation, when the particle number is very small $(N<400)$, the aggregates are also small with lower fractal dimensions (Figures 3 and 4 ). The aggregate grows to a highly assembled three-dimensional aggregation structure with the rising particle numbers; the fractal dimension rises sharply as the particle number increases from 50 to 400 , and then it varies little as the particle number continues to increase. When the particle number is above 1000 , the fractal dimension generally stays at around 2.20, which agrees with the fractal dimension values of representative 3D DLA aggregates [30]. However, the porosity only slightly fluctuates with varying particle numbers because the 3D DLA aggregate has a much bigger packing volume compared with the 2D DLA aggregate. Thus, the optimal particle number for 3D DLA simulation is 1000. For 2D and 3D DLA aggregates, the fractal dimensions generally increase with flocculation time elapsed (rising particle numbers) when $N_{s}<1000$, and then fluctuate little.
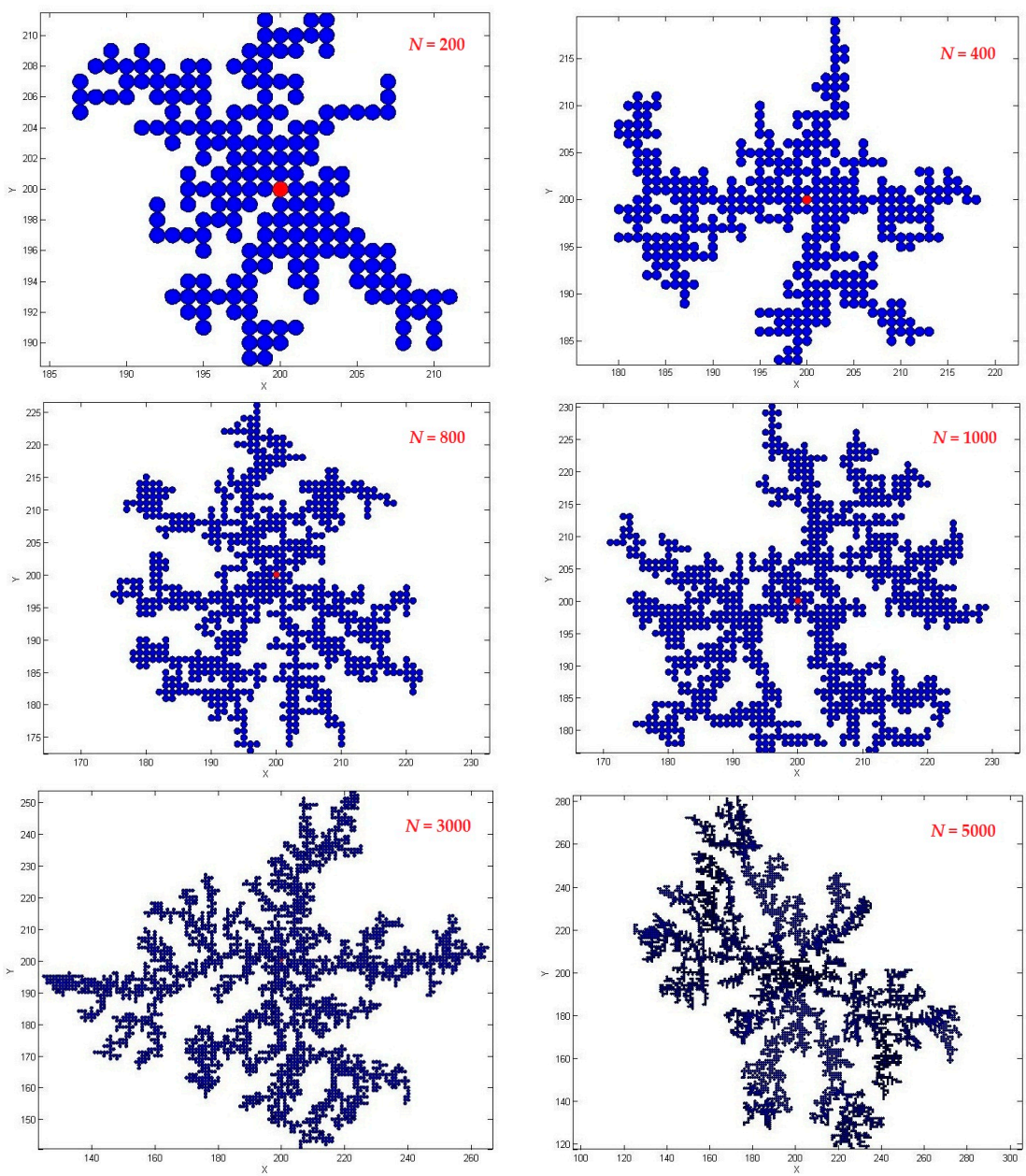

Figure 2. 2D DLA aggregates with versatile particle numbers. 

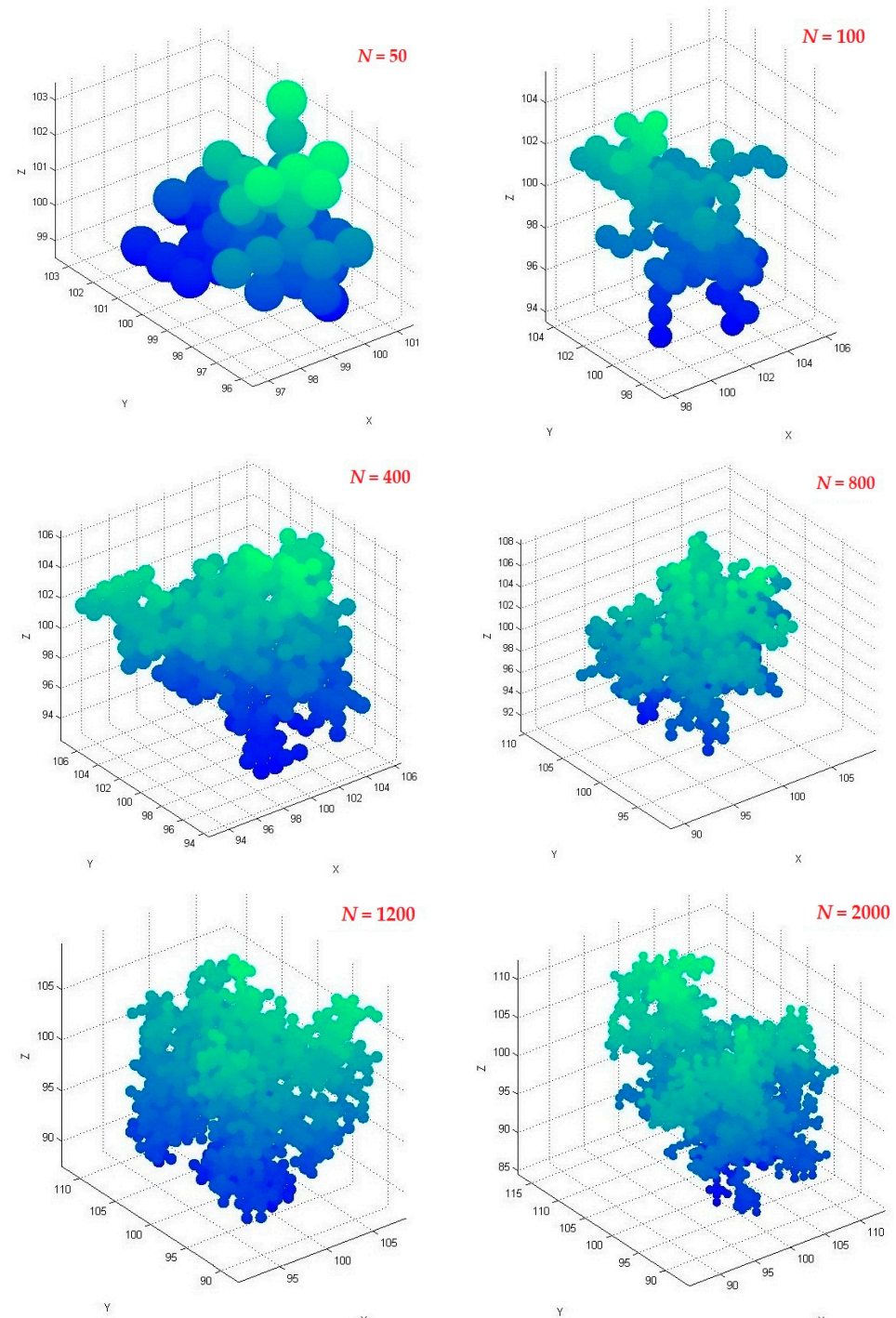

Figure 3. 3D DLA aggregates with versatile particle numbers.
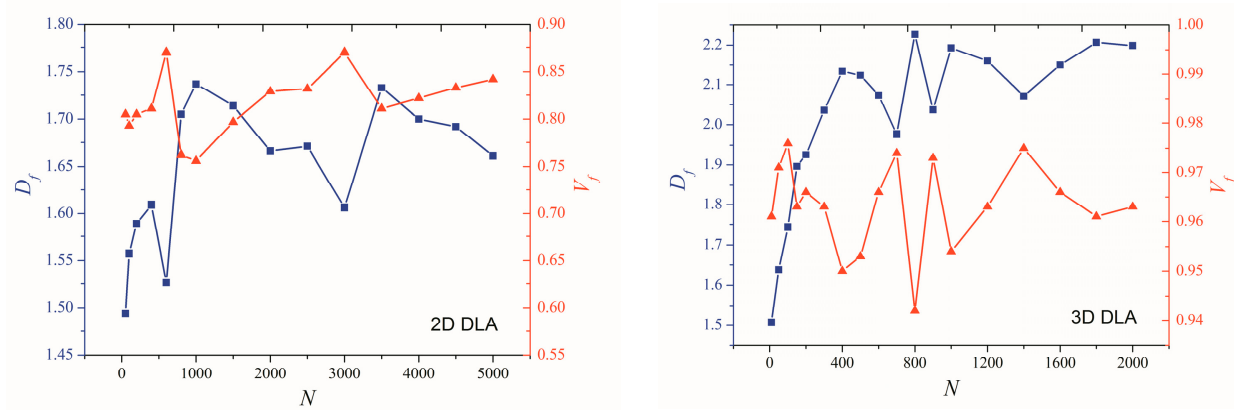

Figure 4. Effect of particle number on the fractal dimension and porosity of 2D and 3D DLA aggregates.

\subsection{Effect of Motion Step Length}

The fractal simulation was implemented with particle numbers of 2000 (2D DLA) and 1000 (3D DLA), a launch radius of $2\left(R_{e}=2\right)$, and motion step lengths varying from 0.5 to 10 ; the effect of motion step length on the fractal features and structures of the DLA aggregates is illustrated in Figures 5 and 6 . 
It is well known that water temperature has a big influence on flocculation processes: High water temperature can accelerate the movement of colloidal particles and hydrolytic processes of flocculants, which contributes to the enhancement of flocculation efficiency. Thus, the motion step length is adopted to denote the water temperature of the practical flocculation processes: the bigger the motion step length, the higher the water temperature. When the motion step length is small $\left(N_{S}<4\right)$, the 2D DLA aggregates present two-dimensional slender dendritic planar structures of typical fractal features with scale-invariant properties, and the 3D DLA aggregates show fairly loose three-dimensional spatial structures. The DLA aggregate gradually grows to an increasingly compact structure as the motion step length rises from 4 to 10 : The 2D DLA aggregate generates a dense cake-like structure of inconspicuous fractal features with higher fractal dimension $\left(D_{f}=1.92\right)$, and the 3D DLA aggregate forms a highly compact ball-like structure with higher fractal dimension of 2.40 when the motion step length approaches 10 . With increasing motion step length, the fractal dimensions gradually increase while the porosities decrease correspondingly (Figure 7). The reason for this is that the smaller the motion step length is, the smaller the particle motion region is, and the released particles can be more easily captured by the external branches of the aggregates, leading to the rapider growth of the aggregate branches with highly slender structures, which contributes to the decrease in fractal dimensions and increase in porosities. Conversely, when the motion step length is bigger, the motion region of the particle becomes larger, and it is more difficult for the aggregate external branches to capture the launched particles that can more easily go into the interior of the aggregate to collide and coagulate with the "seed" particle or clusters. This can eliminate the shielding effect and make the aggregate grow more slowly with less branches to form a highly compact structure, leading to the increase in fractal dimension and decrease in porosity. Thus, the DLA aggerates will grow into increasingly compact objects with inconspicuous fractal features as the water temperature (the motion step length) increases. It should be noted that when the motion step length is equal to $2\left(N_{S}=2\right)$, the DLA aggregate presents prominent fractal structure characteristics.
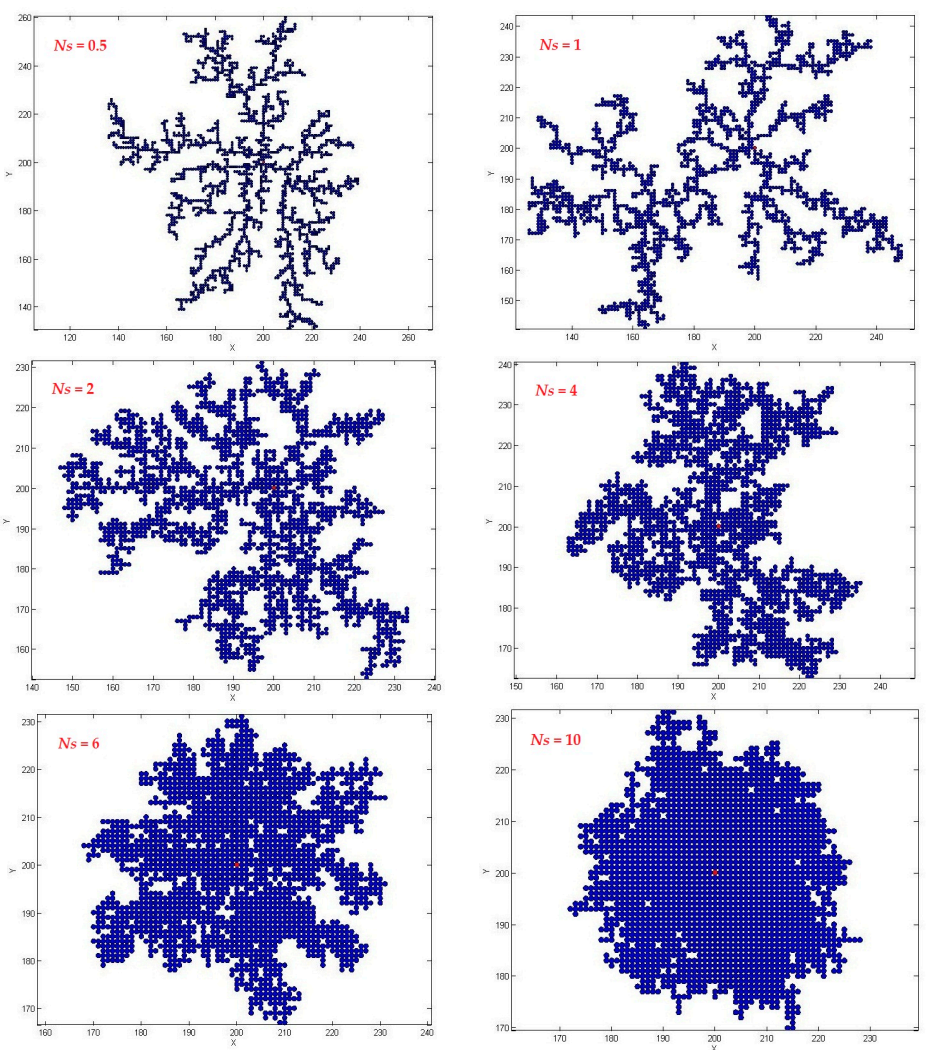

Figure 5. 2D DLA aggregates with different motion step lengths. 

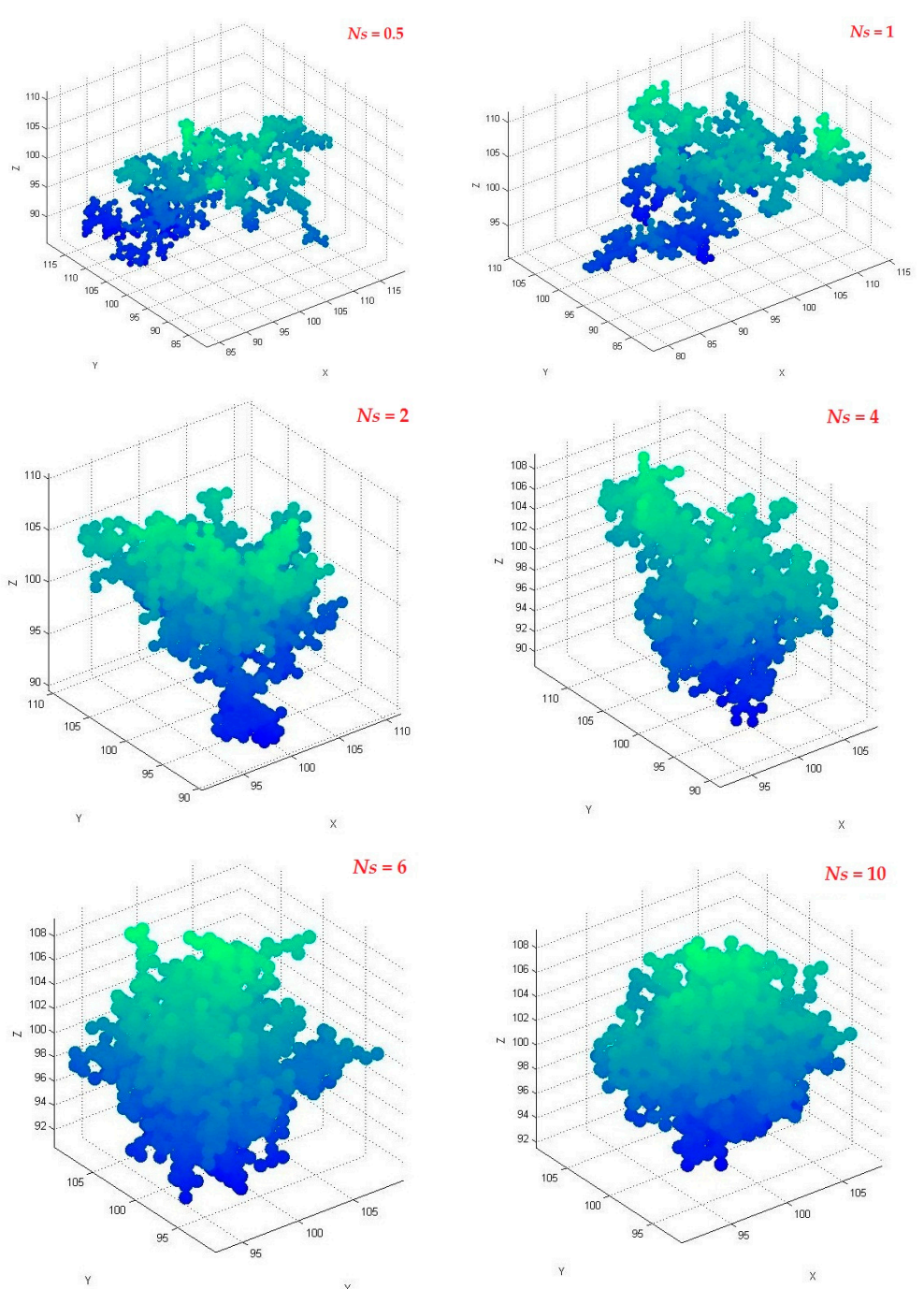

Figure 6. 3D DLA aggregates with different motion step lengths.
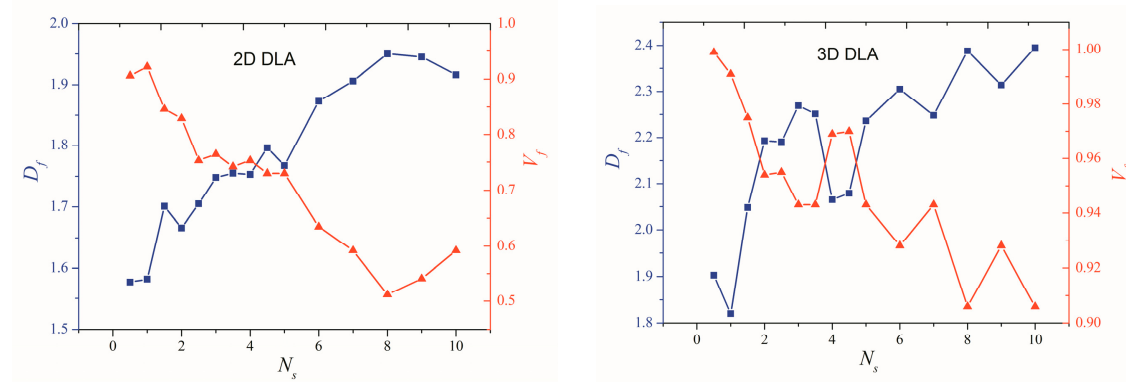

Figure 7. Effect of motion step length on the fractal dimension and porosity of 2D and 3D DLA aggregates.

\subsection{Effect of Launch Radius}

The fractal simulation was performed with particle numbers of 2000 (2D DLA) and 1000 (3D DLA), a motion step length of $2\left(N_{s}=2\right)$, and a versatile launch radius ranging from 1 to 50 . The effect of launch radius on the fractal features and structures of the DLA aggregates is displayed in Figures 8 and 9 . The launch radius can represent the initial particulate concentration of the practical flocculation processes: A bigger launch radius means that the initial particulates in the wastewater are further away 
from each other; that is, the initial particulate concentration is lower. As we can see, the aggregates gradually grow outward with the increasing launch radius. When the launch radius rises from 1 to 50 , the fractal dimensions of the 2D DLA aggregates generally decrease from 1.69 to 1.53 , and the porosities slightly increase from 0.82 to 0.89 ; the fractal dimensions of the 3D DLA aggregates generally drop from 2.24 to 2.01, and the porosities slowly rise from 0.94 to 0.98 (Figure 10). When the launch radius exceeds 10, the fractal dimensions of the 2D DLA aggregates fluctuate between 1.53 and 1.71, and the porosities stay at around 0.82-0.90; the fractal dimensions of the 3D DLA aggregates fluctuate between 2.01 and 2.16, and the porosities stay at $0.96-0.98$. The bigger the launch radius, the larger the motion radius of the particle and, thus, it is easier for the released particles to go outward, resulting in the outward growth of the aggregate as well as the decrease in fractal dimension and increase in porosity. Thus, the DLA aggregates will grow increasingly outward with rising initial particulate concentration (launch radius) accompanying the reduction of fractal dimensions and rise of porosities. It can be concluded that the DLA aggregates display conspicuous fractal features when the launch radius is between 1 and 10 .
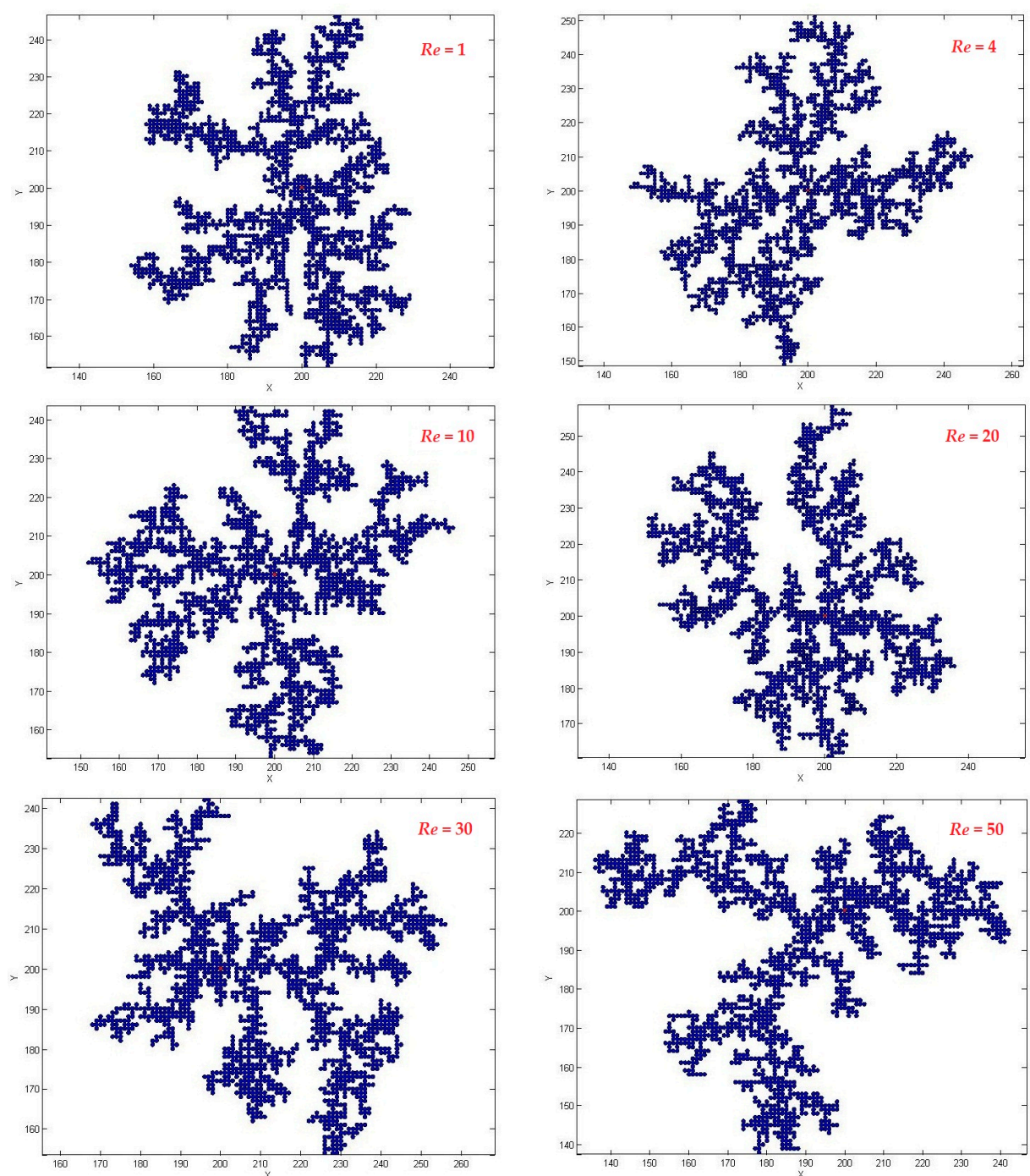

Figure 8. 2D DLA aggregates with various launch radii. 

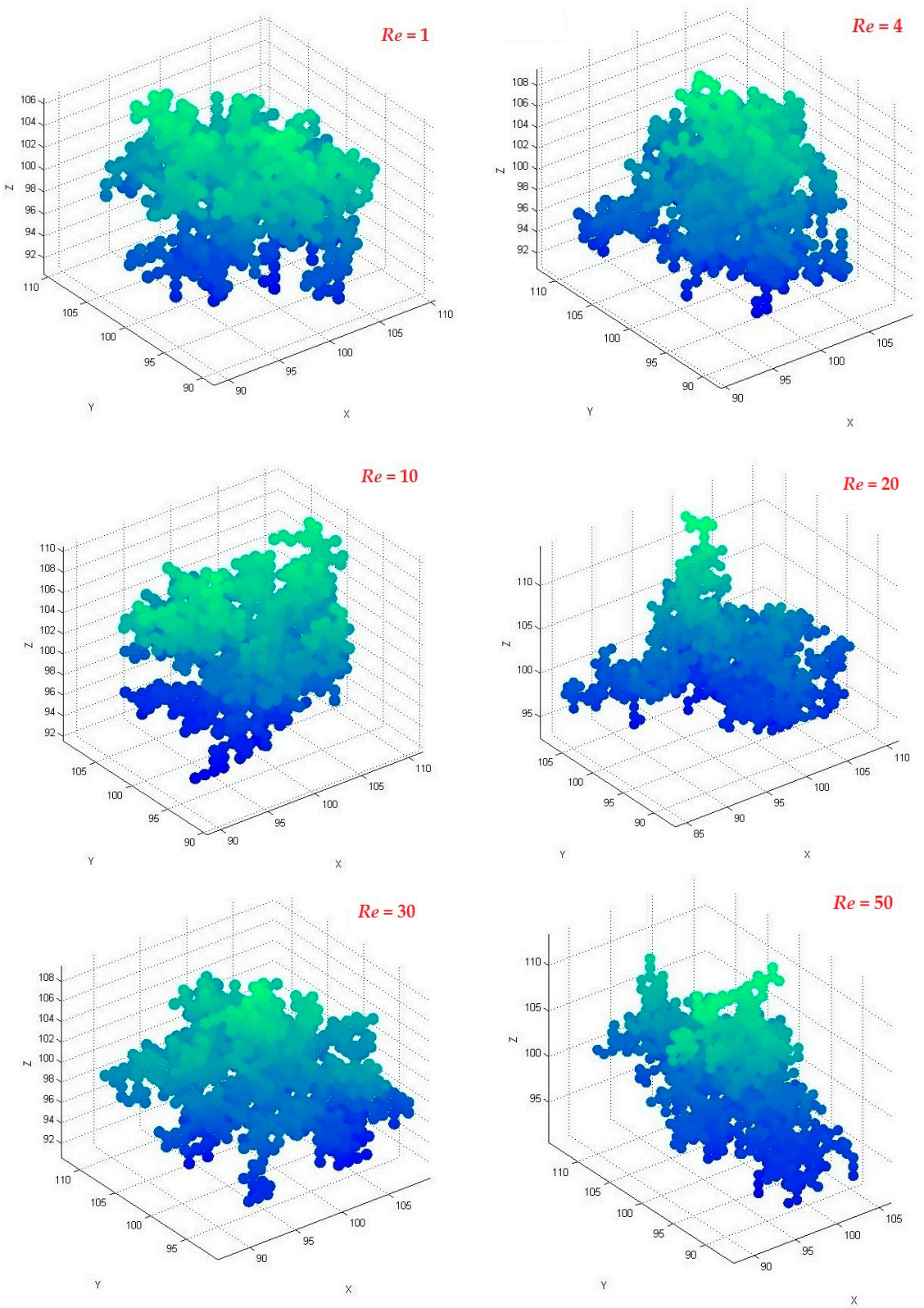

Figure 9. 3D DLA aggregates with various launch radii.
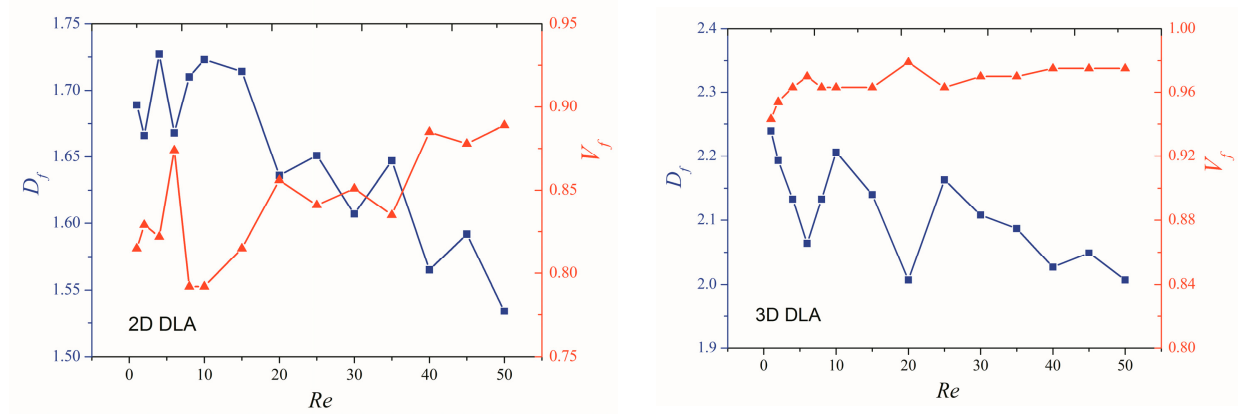

Figure 10. Effect of launch radius on the fractal dimension and porosity of 2D and 3D DLA aggregates. 


\subsection{Effect of Finite Motion Step}

In practical flocculation processes, the movement energy of colloidal particles is limited, and the particles will stop moving if the energy is dissipated. Therefore, finite motion steps were introduced into the DLA model to describe the flocculation processes more accurately. We assume that the random walking particles would cease and become new "seed" particles when their motion steps reach certain given values. The fractal simulation was carried out at particle numbers of 2000 (2D DLA) and 1000 (3D DLA), motion step length of $2\left(N_{s}=2\right)$, and launch radius of $2\left(R_{e}=2\right)$ with various finite motion steps. The effect of the finite motion step on the fractal features and structures of the DLA aggregates is presented in Figures 11 and 12.
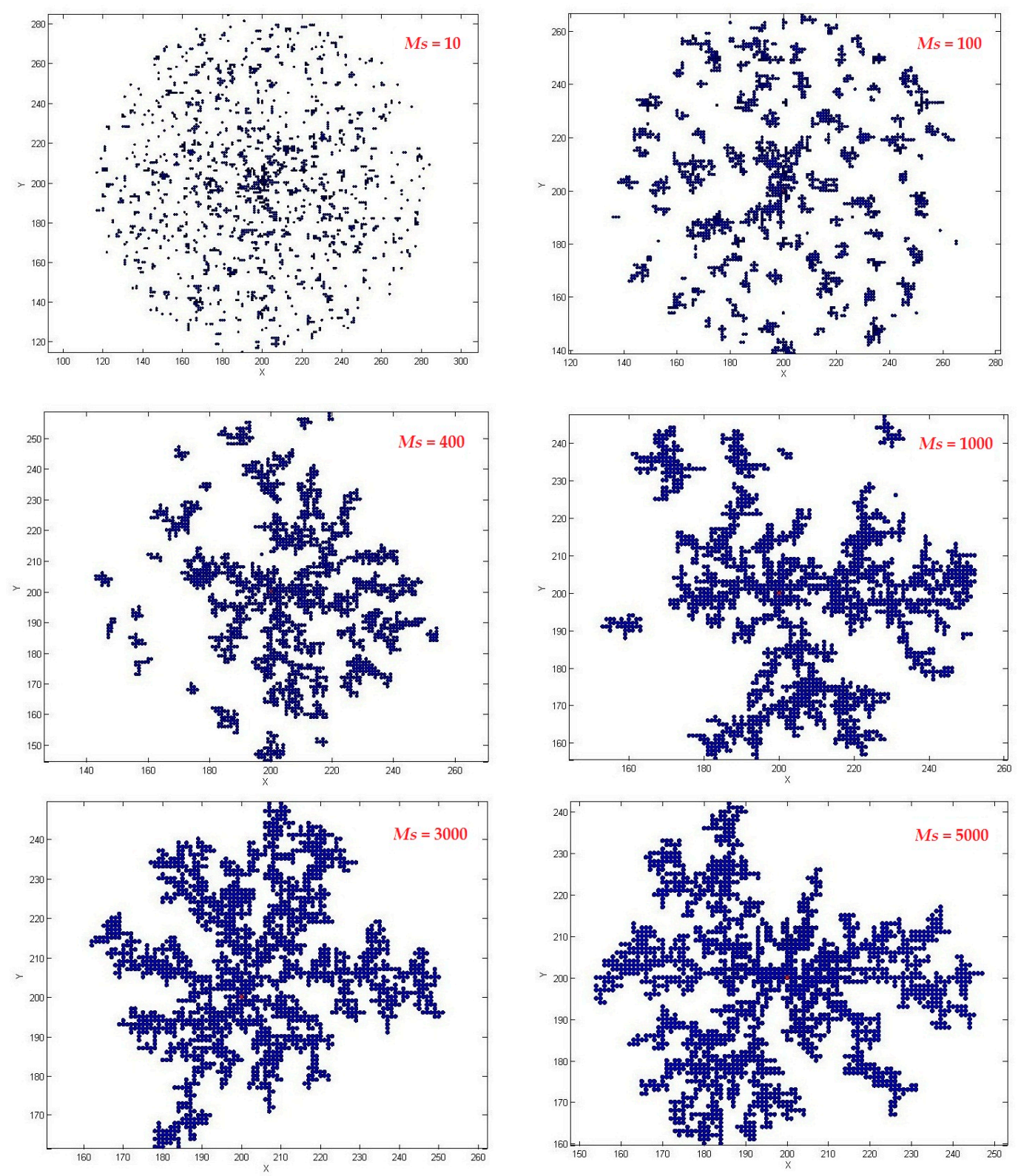

Figure 11. 2D DLA aggregates with diverse finite motion step numbers. 

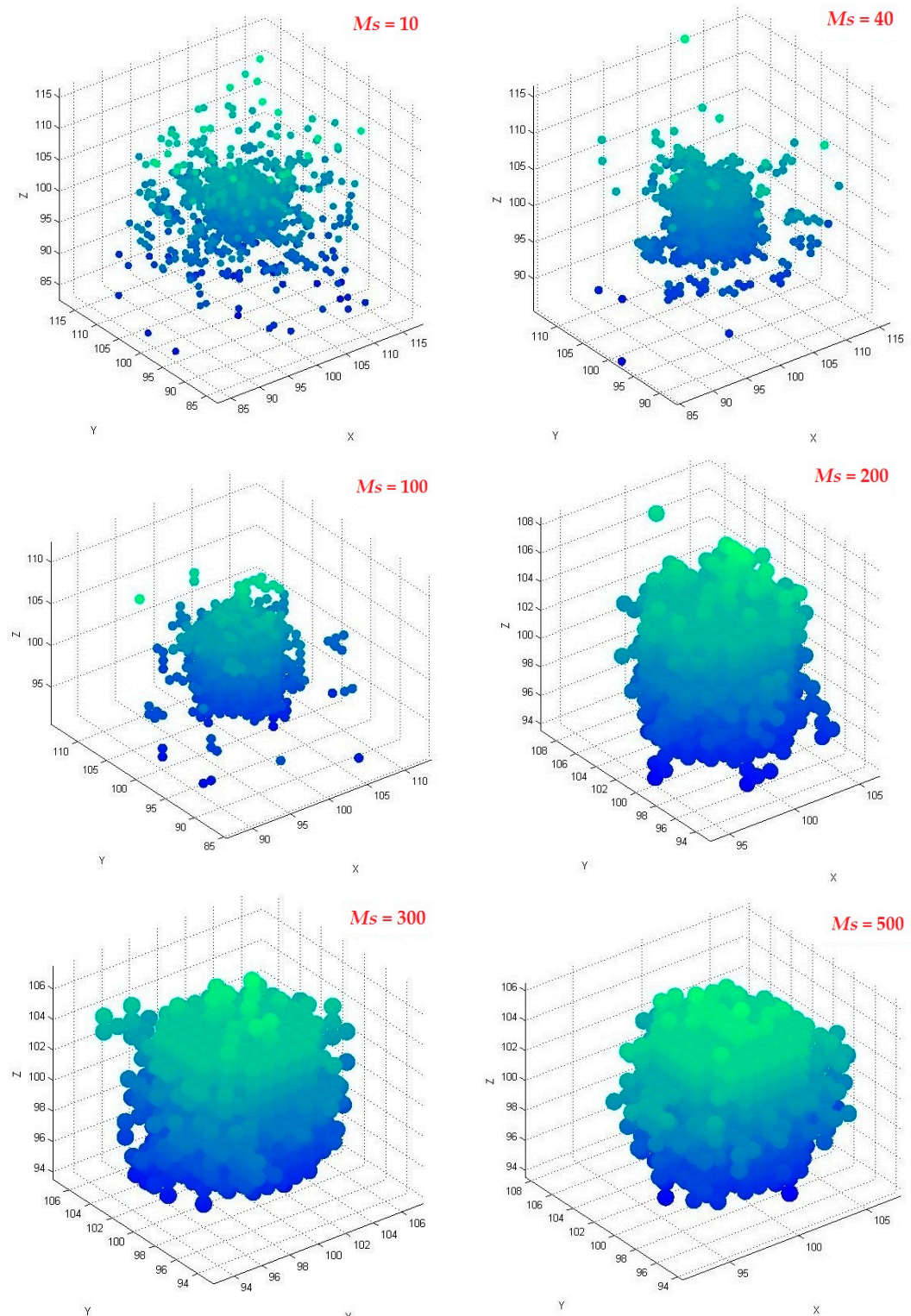

Figure 12. 3D DLA aggregates with diverse finite motion step numbers.

For the 2D DLA simulation, when the finite motion step numbers are below 800 , the aggregates present a highly discrete morphology consisting of numerous separated tiny clusters with lower fractal dimensions $\left(D_{f}<1.60\right)$ and larger porosity $\left(V_{f}>0.87\right)$. They grow increasingly discrete and the fractal dimension reduces rapidly with the gradual decrease of the finite motion step numbers. When $M_{s}$ exceeds 800 (but is less than 3000), the aggregates grow to increasingly compact branched structures with typical scale-invariant features, and fractal dimension between 1.62 and 1.69 (Figure 13). However, the fractal dimension slightly increases to 1.64-1.74 when the finite motion step numbers rise from 3000 to 5000. For the 3D simulation, when the finite motion step numbers are less than 200, the aggregates exhibit a highly scattered spatial structure composed of a few isolated small clusters with lower fractal dimensions $\left(D_{f}<1.95\right)$ and larger porosity $\left(V_{f}>0.88\right)$. They also grow increasingly scattered and the fractal dimension drops sharply with decreasing finite motion step numbers. When $M_{s}$ exceeds 200, the aggregates produce increasingly compact spatial structures, and the fractal dimension stays at 2.00-2.30. 
The smaller the finite motion step is, the lower the particle motion energy is; thus, it is harder for the particles to collide into the "seed" particle or clusters. With the motion energy dissipating away (reaching the maximum motion step), the particles would stop moving and become new "seed" particles, and would generate new tiny clusters after colliding and sticking with other randomly walking particles. This prevents the particles from going into the interior of the aggregates, contributing to the formation of highly discrete or scattered aggregates with lower fractal dimensions and larger porosities. On the contrary, the motion energy of the particles gradually increases with the rising finite motion step and, therefore, it is easier for the particles to collide into the "seed" particle or clusters. The energy of the motion particles may not entirely dissipate away before they coagulate, contributing to the generation of more compact aggregates, leading to the increase in fractal dimensions and the decrease in porosities $[26,27]$.
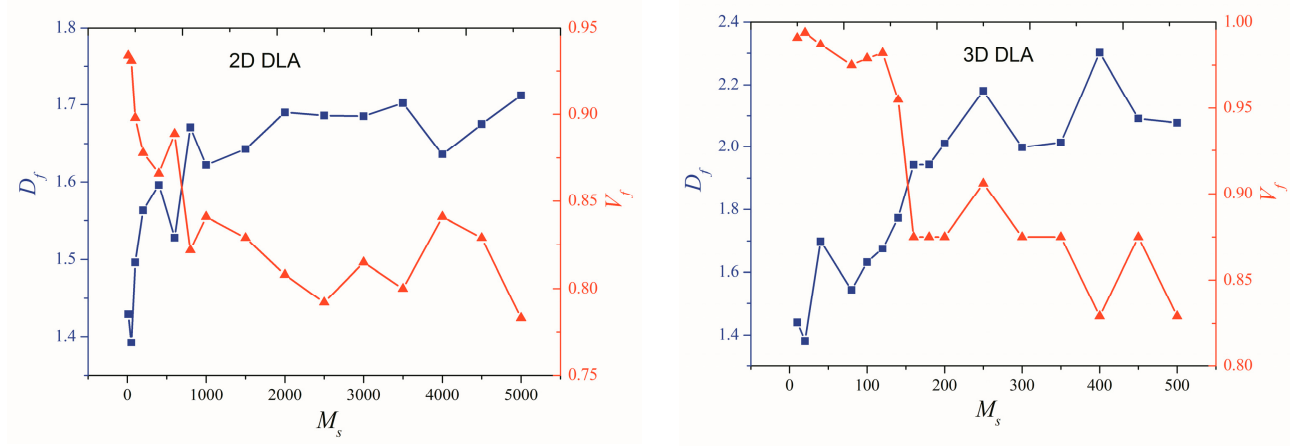

Figure 13. Effect of finite motion step numbers on the fractal dimension and porosity of 2D and 3D DLA aggregates.

\section{Conclusions}

A fractal simulation of flocculation processes using the DLA model with varying particle numbers, motion step lengths, launch radii, and finite motion steps was conducted. The above-mentioned four model parameters all have great influence on the morphologies and structures of the DLA aggregates. For the 2D DLA model, the simulation with too few particles $(<1000)$, too big a motion step length $(>4)$, too large a launch radius $(>10)$, and too small a finite motion step $(<800)$ cannot generate 2D DLA aggregates with clear fractal features and global scale-invariant properties. The 2D DLA simulation with the particle number of $>1000$, motion step length of 1.5-3.5, launch radius of $1-10$, and finite motion step of $>3000$ can form 2D DLA aggregates possessing conspicuous fractal features with fractal dimensions of around 1.70, which is close to the experimental result of 1.66 reported in [10]. For the 3D DLA model, the simulation with too few particles $(<500)$, too big a motion step length $(>4)$, too large a launch radius $(>10)$, and too small a finite motion step $(<200)$ cannot produce 3D DLA aggregates with clear fractal features, either. The 3D DLA simulation with the particle number of $>500$, motion step length of 1.5-3.5, launch radius of 1-10, and finite motion step of $>200$ can form 3D DLA aggregates having apparent fractal features with fractal dimensions of around 2.20, which agrees well with the experimental values of 1.8-2.20 reported in [30] and is considerably higher than that of the 2D DLA aggregates, probably due to the excluded volume effect [7] which means that the centers of the primary particles in three-dimensional space cannot approach each other more closely than the length of the diameter of the primary particle. As reported, the diffusion-limited cluster-cluster aggregation model (DLCA) with fractal dimension of 1.75-1.85 [31-33] can reveal the real flocculation processes better than the DLA model; we intend to conduct further research on fractal simulations of the flocculation processes by using the DLCA model as well.

Author Contributions: Dongjing Liu conducted the simulations and wrote the paper; Weiguo Zhou, Zumin Qiu, and $\mathrm{Xu}$ Song contributed valuable ideas and discussions. 
Conflicts of Interest: The authors declare no conflict of interest.

\section{References}

1. Moghaddam, S.S.; Moghaddam, M.R.A.; Arami, M. Coagulation/flocculation process for dye removal using sludge from water treatment plant: Optimization through response surface methodology. J. Hazard. Mater. 2010, 175, 651-657. [CrossRef] [PubMed]

2. Wu, Y.F.; Liu, W.; Gao, N.Y.; Tao, T. A study of titanium sulfate flocculation for water treatment. Water Res. 2011, 45, 3704-3711. [CrossRef] [PubMed]

3. Gan, L.M.; Yeoh, K.W.; Chew, C.H.; Koh, L.L.; Tan, T.L. Poly(sodium acrylamidoalkanoate) s: Syntheses and solution properties in relation to flocculation study in water treatment. J. Appl. Polym. Sci. 2010, 42, $225-232$. [CrossRef]

4. Jiang, Q.; Logan, B.E. Fractal dimensions of aggregates from shear devices. J. Am. Water Works Assoc. 1996, $88,100-113$.

5. Oles, V. Shear-induced aggregation and breakup of polystyrene latex particles. J. Colloid Interface Sci. 1992, 154, 351-358. [CrossRef]

6. Adachi, Y.; Kobayashi, A.; Kobayashi, M. Structure of colloidal flocs in relation to the dynamic properties of unstable suspension. Int. J. Polym. Sci. 2012, 2012, 1-14. [CrossRef]

7. Adachi, Y.; Kobayashi, M.; Ooi, S. Applicability of fractals to the analysis of the projection of small flocs. J. Colloid Interface Sci. 1998, 208, 353-355. [CrossRef] [PubMed]

8. Matsumoto, T.; Adachi, Y. Effect of ionic strength on the initial dynamics of flocculation of polystyrene latex with polyelectrolyte. J. Colloid Interface Sci. 1998, 204, 328-335. [CrossRef] [PubMed]

9. Kobayashi, M.; Adachi, Y.; Ooi, S. On the steady shear viscosity of coagulated suspensions. J. Soc. Rheol. Jpn. 2000, 28, 143-144. [CrossRef]

10. Sato, D.; Kobayashi, M.; Adachi, Y. Effect of floc structure on the rate of shear coagulation. J. Colloid Interface Sci. 2004, 272, 345-351. [CrossRef] [PubMed]

11. Adachi, Y.; Aoki, K. Restructuring of small flocs of polystyrene latex with polyelectrolyte. Colloids Surf. A Physicochem. Eng. Asp. 2009, 342, 24-29. [CrossRef]

12. Witten, T.A.; Sander, L.M. Diffusion-limited aggregation. Phys. Rev. B Condens. Matter 1983, 27, $5686-5697$. [CrossRef]

13. Meakin, P. Formation of fractal clusters and networks by irreversible diffusion-limited aggregation. Phys. Rev. Lett. 1983, 51, 1119-1122. [CrossRef]

14. Meakin, P. The structure of two-dimensional Witten-Sander aggregates. J. Phys. A Gen. Phys. 1985, 18, L661-L666. [CrossRef]

15. Meakin, P.; Vicsek, T. Diffusion-limited aggregation with radial bias. J. Phys. A Gen. Phys. 1987, 20, L171-L175. [CrossRef]

16. Sander, L.M.; Cheng, Z.M.; Richter, R. Diffusion-limited aggregation in three dimensions. Phys. Rev. B 1983, 27, 5686-5697. [CrossRef]

17. Paterson, L. Diffusion-limited aggregation and two-fluid displacements in porous media. Phys. Rev. Lett. 1984, 52, 1621-1624. [CrossRef]

18. Rangel, R.; Rojas, S. Montecarlo DLA-type simulations of wetting effects in fluid displacement in porous media. Comput. Geosci. 2009, 13, 215-225. [CrossRef]

19. Gentile, F.; Coluccio, M.L.; Toma, A.; Rondanina, E.; Leoncini, M.; Angelis, F.D.; Das, G.; Dorigoni, C.; Candeloro, P.; Fabrizio, E.D. Electroless deposition dynamics of silver nanoparticles clusters: A diffusion limited aggregation (DLA) approach. Microelectron. Eng. 2012, 98, 359-362. [CrossRef]

20. Sun, W.; Wang, W.; Gu, Y.; Xu, X.; Gong, J. Study on the wax/asphaltene aggregation with diffusion limited aggregation model. Fuel 2017, 191, 106-113. [CrossRef]

21. Novotny, M.A.; Tao, R.; Landau, D.P. Relaxation in DLA with surface tension. J. Phys. A Gen. Phys. 1990, 23, 3271-3278. [CrossRef]

22. Tan, Z.J.; Zou, X.W.; Zhang, W.B.; Jin, Z.Z. Influence of particle size on diffusion-limited aggregation. Phys. Rev. E Stat. Phys. Plasmas Fluid. Relat. Interdiscip. Top. 1999, 60, 6202-6205. [CrossRef]

23. Braga, F.L.; Mattos, O.A.; Amorin, V.S.; Souza, A.B. Diffusion limited aggregation of particles with different sizes: Fractal dimension change by anisotropic growth. Phys. A Stat. Mech. Appl. 2015, 429, 28-34. [CrossRef] 
24. Deng, L.; Wang, Y.; Ouyang, Z. Diffusion-limited aggregation with polygon particles. Commun. Theor. Phys. 2012, 58, 895-901. [CrossRef]

25. $\mathrm{Xu}, \mathrm{C}$. A flocculation simulation method based on two-dimensional DLA model. J. Simul. 2014, 2, 19-22.

26. Qiu, Z.; Liu, D.; Zhang, R. Simulation of two-dimensional floc growth using improved DLA model. In Proceedings of the 2011 IEEE 2nd International Conference on Computing, Control and Industrial Engineering, Wuhan, China, 20-21 August 2011; pp. 246-249.

27. Qiu, Z.; Liu, D.; He, Z.; Wu, Y. Simulation of three-dimensional floc growth using improved DLA model. In Proceedings of the 2011 IEEE 2nd International Conference on Computing, Control and Industrial Engineering, Wuhan, China, 20-21 August 2011; pp. 250-253.

28. Scarnato, B.V.; Vahidinia, S.; Richard, D.T.; Kirchstetter, T.W. Effects of internal mixing and aggregate morphology on optical properties of black carbon using a discrete dipole approximation method. Atmos. Chem. Phys. 2013, 13, 5089-5101. [CrossRef]

29. Radney, J.G.; You, R.; Ma, X.; Conny, S.M.; Zachariah, M.R.; Hodges, J.T.; Zangmeister, C.D. Dependence of soot optical properties on particle morphology: Measurements and model comparisons. Environ. Sci. Technol. 2014, 48, 3169-3176. [CrossRef] [PubMed]

30. Serra, T.; Casamitjana, X. Structure of the aggregates during the process of aggregation and breakup under a shear flow. J. Colloid Interface Sci. 1998, 206, 505-511. [CrossRef] [PubMed]

31. Wu, H.; Lattuada, M.; Morbidelli, M. Dependence of fractal dimension of dlca clusters on size of primary particles. Adv. Colloid Interface Sci. 2013, 195-196, 41-49. [CrossRef] [PubMed]

32. Heinson, W.R.; Sorensen, C.M.; Chakrabarti, A. Does shape anisotropy control the fractal dimension in diffusion-limited cluster-cluster aggregation? Aerosol Sci. Technol. 2010, 44, 1-4. [CrossRef]

33. Heinson, W.R.; Chakrabarti, A.; Sorensen, C.M. Divine proportion shape invariance of diffusion limited cluster-cluster aggregates. Aerosol Sci. Technol. 2015, 49, 786-792. [CrossRef]

(C) 2017 by the authors. Licensee MDPI, Basel, Switzerland. This article is an open access article distributed under the terms and conditions of the Creative Commons Attribution (CC BY) license (http://creativecommons.org/licenses/by/4.0/). 